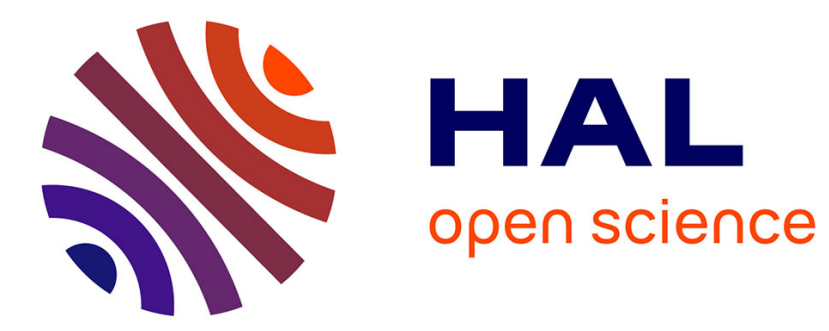

\title{
Blazed binary subwavelength gratings with efficiencies larger than those of conventional échelette gratings
}

Philippe Lalanne, Simion Astilean, Pierre Chavel, Edmond Cambril, Huguette

\section{Launois}

\section{- To cite this version:}

Philippe Lalanne, Simion Astilean, Pierre Chavel, Edmond Cambril, Huguette Launois. Blazed binary subwavelength gratings with efficiencies larger than those of conventional échelette gratings. Optics Letters, 1998, 23 (14), pp.1081-1083. 10.1364/OL.23.001081 • hal-00877238

HAL Id: hal-00877238

https://hal-iogs.archives-ouvertes.fr/hal-00877238

Submitted on 28 Oct 2013

HAL is a multi-disciplinary open access archive for the deposit and dissemination of scientific research documents, whether they are published or not. The documents may come from teaching and research institutions in France or abroad, or from public or private research centers.
L'archive ouverte pluridisciplinaire HAL, est destinée au dépôt et à la diffusion de documents scientifiques de niveau recherche, publiés ou non, émanant des établissements d'enseignement et de recherche français ou étrangers, des laboratoires publics ou privés. 


\title{
Blazed binary subwavelength gratings with efficiencies larger than those of conventional échelette gratings
}

\author{
Philippe Lalanne, Simion Astilean, ${ }^{*}$ and Pierre Chavel \\ Laboratoire Charles Fabry de l'Institut d'Optique, Centre National de la Recherche Scientifique, B.P. 147, F-91403 Orsay Cedex, France
}

Edmond Cambril and Huguette Launois

Laboratoire de Microstructures et de Microélectronique, Centre National de la Recherche Scientifique, 196 Avenue Henri Ravera, B.P. 107, F-92225 Bagneux, France

Received March 24, 1998

\begin{abstract}
We introduce a new structural cutoff beyond which subwavelength gratings cease to behave as homogeneous media and discuss its effects on the proper selection of the sampling periods of subwavelength diffractive elements. According to this analysis, a $3 \lambda$-period blazed binary grating composed of square pillars is designed for $\mathrm{He}-\mathrm{Ne}$ operation and is fabricated by etching of a $\mathrm{TiO}_{2}$ layer deposited upon a glass substrate. Its firstorder measured diffraction efficiency is $12 \%$ larger than the theoretical efficiency of an ideal blazed échelette grating in glass with the same period. (C) 1998 Optical Society of America

OCIS codes: $\quad 050.1380,050.1970,050.1950$.
\end{abstract}

Because of the analogy between subwavelength periodic structures and homogeneous media, binary surface-relief diffractive elements, composed of subwavelength microstructures carefully arranged and etched in a transparent material, may exhibit high diffraction efficiencies. ${ }^{1,2}$ Such elements simulate continuous profiles: The local fraction of matter removed is related through effective-medium theory to the local effective index. With the recent progress of nanofabrication technologies, these elements can now be manufactured ${ }^{3-7}$ for operation in the visible region of the spectrum. In general, the sampling period, or, equivalently, the distance between two adjacent microstructures of the diffractive element, is chosen such that only one transmitted and one reflected order propagate in the substrate and in the incident medium. This choice amounts to selecting a sampling period smaller than the cutoff, defined as the period below which all orders, except the zero orders, are evanescent. For a given angle of incidence the cutoff can be obtained by straightforward use of the grating equation; in what follows, it is denoted $\Lambda_{C}$. It is noteworthy that the cutoff value depends not at all on the grating microstructure geometry but on the refractive indices of the incident medium and of the substrate and on the angle of incidence. Recently it was proposed ${ }^{5}$ to use sampling periods larger than the cutoff to relax fabrication constraints. In this Letter we discuss quantitatively how to select the sampling period properly and introduce a new cutoff value beyond which the analogy between subwavelength gratings and homogeneous media is no longer valid. This new cutoff, $\Lambda_{S}$, is called the structural cutoff to emphasize that it is intrinsic in the grating microstructure geometry. At the same time, we use this quantitative analysis to design a $3 \lambda$-period grating composed of square subwavelength pillars operating for unpolarized light and report on experimental efficiencies larger than those achieved theoretically by conventional échelette gratings in glass with the same period.

0146-9592/98/141081-03\$15.00/0
Recently we proposed ${ }^{7}$ using high-index materials for the fabrication of subwavelength diffractive components operating in the visible. We showed that this approach reduces fabrication constraints and increases the diffraction efficiency; for $\mathrm{He}-\mathrm{Ne}$ operation under TM polarization, we reported an $83 \%$ efficiency component composed of one-dimensional grooves etched in a $\mathrm{TiO}_{2}$ film $\left(n_{h}=2.30\right)$ coated upon a glass substrate $\left(n_{g}=1.52\right)$. Hereafter we consider again blazed binary gratings etched in a $\mathrm{TiO}_{2}$ film but composed of square pillars for operation under unpolarized light at $633 \mathrm{~nm}$. Normal incidence from air is assumed throughout, and the wavelength of the incident plane wave is $633 \mathrm{~nm}$. We now focus on how to select the sampling period. For that purpose we first consider a grating composed of a square array of square pillars. We define the fill factor $f$ as the ratio of the pillar width to the grating period and denote by $x$ and $y$ the periodicity axes of the grating and by $z$ the normal to the grating boundaries. We look for modes that are propagating inside the grating region along the $z$ direction. The $z$ dependence of the modes is expressed as $\exp \left(j k_{0} n z\right)$, where $k_{0}$ is the wave-vector modulus of the incident plane wave in vacuum. The values $n$ of the normalized wave-vector amplitudes are computed from Bloch wave theory by expansion of the electromagnetic fields along the $x$ and $y$ directions on a Fourier basis. The computation consists of solving the eigenproblem of Ref. 10 and retaining the eigenvalues. To illustrate the procedure we consider a grating period of $380 \mathrm{~nm}$ (this value is also considered for fabrication hereafter). The dotted curves in Fig. 1 represent the positive values of $n$ for all the propagating modes. Roughly speaking, we found that only one mode propagates in the structure for $f<0.5$, two modes propagate for $0.5<f<0.75$, and three for $f>0.75$. The uppermost dotted curve corresponds to the fundamental mode; under normal incidence, its associated eigenvalue is equal to the grating effective index. As shown in Ref. 8, only this mode propagates in the structure for infinitely small period-to-wavelength ratios. 


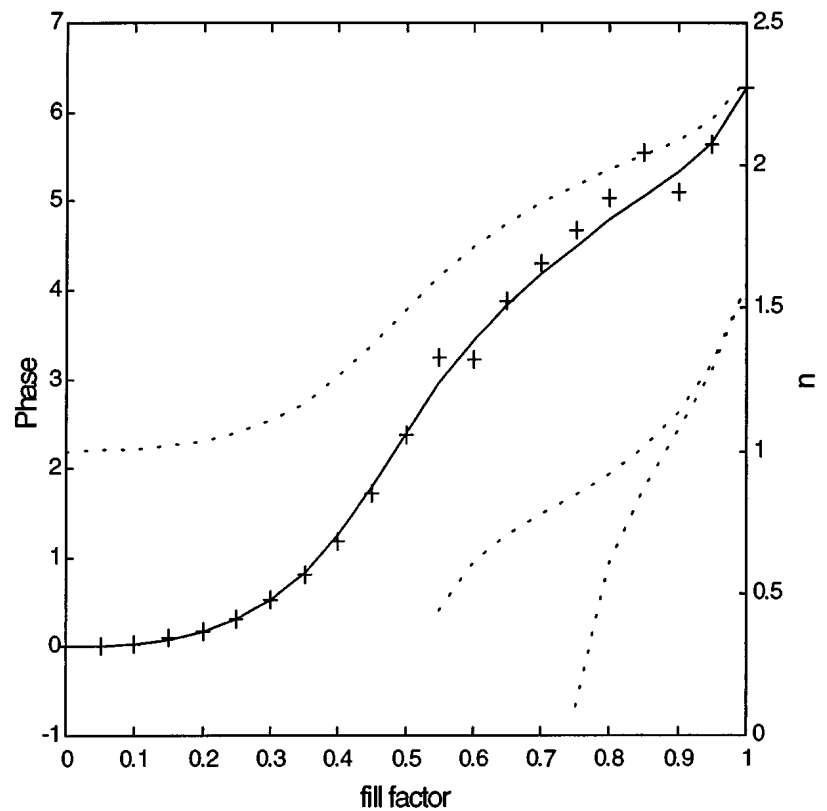

Fig. 1. Crosses, phases of the transmitted zeroth order for a 487-nm-thick grating composed of a 380-nm-period array of square pillars etched in $\mathrm{TiO}_{2}$. Dotted curves, $n$ values of all the propagating modes supported by the biperiodic structure. The uppermost curve ( $n$ varies from 1 to 2.3 ) corresponds to the grating effective index. Numerical results were obtained by the modal method of Ref. 10 with square truncation (17 orders are retained along each axis). Solid curve, phases of a plane wave transmitted through a 487-nm-thick homogeneous dielectric film whose refractive index is equal to the $n$ value of the fundamental mode.

The other eigenvalues (lower dotted curves) correspond to propagating modes that are evanescent at small periods.

The solid curve in Fig. 1 shows the phase changes of a plane wave transmitted through a homogeneous dielectric film whose refractive index is equal to the $n$ value of the fundamental mode. The Airy formula ${ }^{9}$ was used for the computation, with the assumption that the film is coated upon a glass substrate. The film thickness was chosen to be $487 \mathrm{~nm}$ to accommodate a phase range of $0-2 \pi$. Crosses represent the phase of the transmitted zeroth order of a grating with the same 487-nm thickness. The computation was performed with the electromagnetic theory of Ref. 10 for various fill factors. A comparison of the solid curve and the crosses indicates that, when only one mode propagates $(f<0.5)$, almost no difference is found between the two phases. For $f>0.5$, a deviation is observed. It is noteworthy that, whereas the phase associated with the thin-film computation is smoothly increasing with $f$, that obtained with electromagnetic theory exhibits chaotic behavior and is not a monotonic function of $f$. From this simple analysis we conclude that periodic structures (with subcutoff or supercutoff periods such as those considered in Ref. 5) that support more than one propagating mode do not mimic homogeneous media. When only one mode propagates in a grating (all the others are evanescent), this mode travels backward and forward between the two grating boundaries in the same way as multiple beam interference occurs in a thin film. Consequently the zeroth-order reflected and transmitted amplitudes are approximately those of a thin film with a refractive effective index equal to the normalized wave-vector modulus $n$ of this mode. ${ }^{8}$ Clearly, this situation is no longer valid when evanescent modes cannot be ignored (as occurs for small grating depths; see Ref. 11) or if more than one mode propagates in the grating region. For any fraction of removed material and for a given microstructure geometry, the period (or structural cutoff) below which a dielectric grating supports only one propagating mode (this mode can be polarization dependent) for a given incidence angle is a relevant parameter that has to be taken into account in selection of an appropriate sampling period. The ratio $\Lambda_{s} / \lambda$ depends on the angle of incidence, the microstructure geometry, and the permittivity modulation. From this analysis we do not conclude that the structural cutoff is a definite limit that must not be exceeded; rather, it is an important parameter that one needs to consider when selecting the sampling period. The following design will illustrate our purpose.

We now consider the design of a prismlike grating composed of subwavelength square pillars etched in a $\mathrm{TiO}_{2}$ film coated upon glass for operation at $633 \mathrm{~nm}$. Grating period $\Lambda_{x}$ along the $x$ axis is chosen to be $3 \lambda$. This period corresponds to nearly $20^{\circ}$ deflection in air. Grating period $\Lambda_{y}$ along the $y$ axis is equal to the sampling period. Its value is discussed below. Because of the high index modulation, 2.3/1, we found that the structural cutoff $\Lambda_{s}$ is approximately equal to $270 \mathrm{~nm}$, a value much smaller than $\Lambda_{c}\left(\Lambda_{c}=\lambda / n_{g} \approx 416 \mathrm{~nm}\right)$. Because severe fabrication problems would be encountered with a 270-nm sampling period, we decided to select a larger period $\left(\Lambda_{y}=380 \mathrm{~nm}\right)$, a value much larger than $\Lambda_{s}$ but smaller than $\Lambda_{c}$. For this value, $\Lambda_{x}$ is divided into exactly five sampling periods $\left(\Lambda_{x}=5 \Lambda_{y}\right)$. Following our previous analysis, a natural choice would be to restrict the design to $0<f<0.5$ because only one mode propagates in this interval (see Fig. 1). However, doing so would restrict the maximum available effective index to only 1.5 , and the benefit expected from the use of high-index material would be null. We made a compromise and limited the design to $0<f<0.8$. This somewhat arbitrary choice was motivated by the facts that in this case the maximum available effective index is increased to approximately 2 and that the solid curve and the crosses are nearly identical for $0<f<0.8$ in Fig. 1. Using the uppermost dotted curve of Fig. 1 as a lookup table, we selected five fill factors $(0.31,0.47,0.53,0.65$, and 0.77 ) for the five pillars, corresponding to five effective indices $n_{\text {eff }}$ varying linearly in the interval $[1,2]$. We varied the grating depth and maximized the diffraction efficiency into the first order. For a 545-nm depth, we obtained $77 \%$. This optimization was performed for normal incidence from air, for unpolarized light, and for 25 and 7 retained orders along the $x$ and $y$ axes, respectively. Finally, we optimized the five pillar-center locations along the $x$ axis, using a gradient-descent algorithm and keeping the fill factors, except that of the largest pillar, fixed. We ended up 


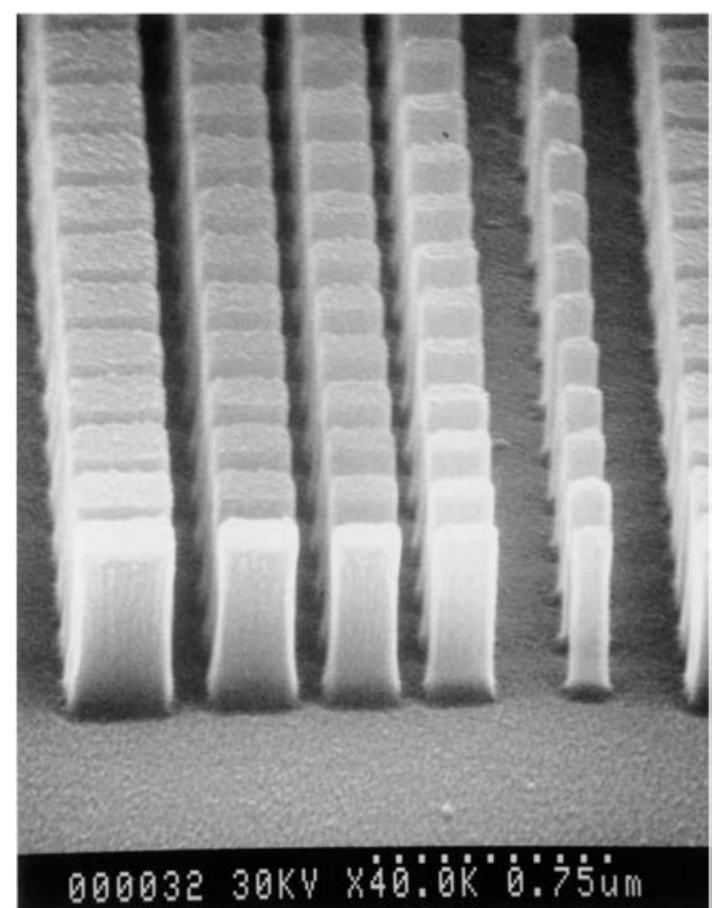

Fig. 2. Scanning-electron micrograph of the blazed binary subwavelength grating. The horizontal period (along the $x$ axis) is $1.9 \mu \mathrm{m}$, and the period in the perpendicular direction ( $y$ axis) is equal to the sampling period (380 $\mathrm{nm}$ ). The maximum pillar aspect ratio is 4.6 .

with a diffraction efficiency of $82 \%$. For TE and TM polarizations (electric field parallel and perpendicular to the $y$ direction), the first-order efficiencies were $82.4 \%$ and $81.7 \%$, respectively.

Grating fabrication involves electron-beam writing in a $150-\mathrm{nm}$-thick poly(methyl methacrylate) layer, lift-off with a nickel mask, and reactive ion etching with a $\mathrm{SF}_{6}(1 / 2) / \mathrm{CH}_{4}(1 / 2)$ gas mixture. Details are given in Ref. 7. The grating pattern was written over a $209 \mu \mathrm{m} \times 229 \mu \mathrm{m}$ area. The writing process lasted 4 min. Larger areas are manufacturable for longer $e$-beam exposures. A scanning-electron photograph of the grating is shown in Fig. 2. On the horizontal scale ( $x$ axis), one grating period $\left(\Lambda_{x}=1.9 \mu \mathrm{m}\right)$ is shown. The grating was tested with a $\mathrm{He}-\mathrm{Ne}$ laser at normal incidence from air. The laser beam waist was focused with a lens of 45-mm focal length. We estimate that more than $99 \%$ of the incoming light passes through the grating aperture. Examination of the farfield pattern revealed little scattering. We estimated that less than $4 \%$ of the light is reflected into the different orders, a small value that is due to the antireflection properties of the grating. We determined the diffraction efficiencies by measuring the powers of the diffracted beams and dividing them by the power of the incident beam. We corrected the measurements by including the Fresnel losses at the back side of the glass substrate. As predicted by theory, we observed that, under normal incidence, the first-order diffraction efficiency is practically independent of polarization; we found $77 \%$ and $78 \%$ for TE and TM polarizations, respectively, values close to those predicted by electro- magnetic theory. Five orders are transmitted through the back side of the glass substrate; four others are totally reflected in the substrate. The highest efficiency $(\approx 7 \%)$ of the five transmitted orders (except for the first one) was observed for the second order and for TE polarization. This measurement is consistent with numerical computations that predict a $5 \%$ efficiency in this case.

To our knowledge this grating exhibits the highest efficiency yet reported for diffractive components composed of two-dimensional subwavelength features and operating with unpolarized visible light. Moreover, it is noteworthy that the maximum diffraction efficiency achieved by a standard blazed échelette grating with a $3 \lambda$ period is $66.5 \%$, a theoretical value $12 \%$ smaller than that obtained experimentally in the research reported here. This efficiency, computed with electromagnetic theory, holds for a grating in glass $\left(n_{g}=1.52\right)$, with an optimized grating depth slightly larger than $\lambda /\left(n_{g}-1\right)$, and for unpolarized light at normal incidence from air. Moreover, simple designs performed for period-to-wavelength ratios in the interval $[2,8]$ but for a sampling period equal to the structural cutoff $\left(\Lambda_{y}=\Lambda_{s}\right.$ and $\Lambda_{x}=\mathrm{N} \Lambda_{s} ; N$ an integer) show that blazed binary subwavelength gratings composed of square pillars in $\mathrm{TiO}_{2}$ consistently outperform conventional blazed échelette gratings in glass. For instance, a diffraction efficiency as high as $87 \%$ was obtained without refinements of the pillar-center location for a $3 \lambda$ grating period.

This research was supported by the European Community under the RODCI Mel-Ari program. The authors are grateful to Jean Landreau and Alain Carenco of the Centre National des Etudes de Télécommunications for coating the $\mathrm{TiO}_{2}$ film.

*Permanent address, Department of Optics and Spectroscopy, Faculty of Physics, Babes-Bolyai University, 3400 Cluj, Napoca, Romania. During the course of this research S. Astilean was a visiting scientist at the Institut d'Optique; he is pleased to acknowledge financial support from NATO.

\section{References}

1. W. Stork, N. Sreibl, H. Haidner, and P. Kipfer, Opt. Lett. 16, 1921 (1991).

2. W. M. Farn, Appl. Opt. 31, 4453 (1992).

3. F. T. Chen and H. G. Craighead, Opt. Lett. 20, 121 (1995).

4. M. E. Warren, R. E. Smith, G. A. Vawter, and J. R. Wendt, Opt. Lett. 20, 1441 (1995).

5. F. T. Chen and H. G. Craighead, Opt. Lett. 21, 177 (1996).

6. J. M. Miller, N. de Beaucoudrey, P. Chavel, E. Cambril, and H. Launois, Opt. Lett. 21, 1399 (1996).

7. S. Astilean, Ph. Lalanne, P. Chavel, E. Cambril, and H. Launois, Opt. Lett. 23, 552 (1998).

8. Ph. Lalanne and D. Lemercier-Lalanne, J. Mod. Opt. 43, 2063 (1996).

9. M. Born and E. Wolf, Principles of Optics, 6th ed. (Macmillan, New York, 1964), Chap. 1, p. 62.

10. L. Li, J. Opt. Soc. Am. A 14, 2758 (1997).

11. Ph. Lalanne and D. Lemercier-Lalanne, J. Opt. Soc. Am. A 14, 450 (1997). 